\title{
Specific Smell Dysfunctions in Relapsing-Remitting Multiple Sclerosis
}

\author{
Valentina Parma ${ }^{1}$, Camilla Tornasi ${ }^{1}$, Paola Grossi ${ }^{2}$, Matteo Atzori², Paola Perini ${ }^{2}$, Massimiliano Calabrese ${ }^{2}$, Roberto Tirindelli ${ }^{3}$, Paolo Gallo ${ }^{2}$ \\ and Umberto Castiello ${ }^{1 *}$
}

${ }^{1}$ Department of General Psychology, University of Padua, Via Venezia, 8 - 35131 Padua, Italy

${ }^{2}$ Department of Neuroscience, University of Padua, Via Giustiniani, 5 - 35128 Padua, Italy

${ }^{3}$ Department of Neuroscience, University of Parma, Via Volturno, 39 - 43100 Parma, Italy

\begin{abstract}
The present study assessed odour threshold, discrimination and identification in relapsing-remitting MS (RRMS) patients. We administered the Sniffin' Sticks Extended Test (Burghart Messtechnik GmbH, Wedel, Germany) to 50 RRMS female patients and to 50 matched control subjects. Also, the number and the volume of MRI-visible demyelinating plaques within the inferior frontal and temporal lobes (IFTL complex), were quantified in a patient subgroup. The results indicated that up to $34 \%$ of the RRMS sample exhibited hyposmia, but none of the MS patients could be considered functionally anosmic. Odour identification and general olfactory performance significantly decreased with respect to the patients' age. No significant correlations between olfactory scores and the number and the volume of plaques within the IFTL complex were found. These findings suggest that some specific forms of olfactory dysfunction do exist in MS patients and that the correlation between olfactory dysfunctions and structural brain damage might not be as strict as previously suggested.
\end{abstract}

Keywords: Multiple sclerosis; Olfaction; Magnetic Resonance Image; Sniffin' Sticks

\section{Introduction}

A controversy within the neurological sciences concerns the presence of olfactory dysfunction in multiple sclerosis (MS) [1,2], the most common chronic disabling neurological disease in young adults, with particular reference to the female population [3]. Whereas some investigators reported that a decrease in odour perception can be one of the symptoms experienced by MS patients [4-10], others have failed to demonstrate any olfactory alterations in MS at all [11-13].

Preliminary evidence in favour of an olfactory dysfunction in MS patients comes from Wender and Szmeja [10], who reported an odour identification dysfunction in 18 out of 52 patients, i.e., 35\% of the examined population. Subsequently, Pinching [9] administered a suprathreshold odour identification task and noticed the presence of anosmia or hyposmia in 10 out of 22 MS patients (i.e., 45.4\%). More recent and compelling demonstrations of altered olfactory perception in MS have been reported in a series of studies using an objective and highly reliable standardized psychophysical test of olfactory functions, the University of Pennsylvania Smell Identification Test (UPSIT) [14-16] or a modified version of the same test [17-19]. In particular, Doty and colleagues[16] found that $23 \%$ of 31 patients obtained UPSIT scores significantly lower than the controls, a result which was confirmed in further studies by the same group which revealed an even higher percentage (38.5\%) of microsmic MS patients $[5,6]$.

Other studies, however, failed to observe olfactory deficits in MS patients. For instance, Ansari [20] using serial binary dilutions of amyl acetate and nitrobenzene to evaluate odour detection thresholds, did not find any difference in the olfactory performance of MS patients compared with age- and sex-matched healthy controls. Similarly, MS patients scored as high as healthy controls in two further studies using the UPSIT $[11,13]$.

Previous research has also considered the MRI approach, which implies the correlation of olfactory test scores with the in vivo quantitative assessment of focal demyelinating areas (i.e., plaques) within primary olfactory regions by means of high-resolution magnetic resonance imaging (MRI). A strong negative correlation between the olfactory test scores (i.e., UPSIT) and the number of demyelinating plaques within the inferior frontal and temporal lobe (IFTLs) regions, which are involved in olfaction, has been reported [5,7]. Such correlations were not evident in brain regions unrelated to olfaction [5]. Similar evidence was reported in a study in which a robust correlation between a T2 lesion load within the IFTLs complex and smell loss was found [19]. From the above brief literature review it emerged that a clear consensus on this matter has yet to be reached. One of the factors which might have contributed to such controversial results is the nature of the olfactory test that was administered. Indeed, different - sometimes non-standardized tests - were used $[9,10,20]$ and only odour identification was chiefly considered $[4,6-8,13,17-20]$. Also, even when the same test for the evaluation of odour identification was utilized (i.e., the UPSIT), the opposite results were found $[5,13]$. To date, the effect of MS on other measures of olfactory performance, such as odour threshold and discrimination, remain largely unknown.

Therefore the main aim of the present study was to elucidate whether RRMS patients are affected by a loss of smell, either global or task specific. A secondary aim was to evaluate the possible correlation between olfactory dysfunctions and the presence of demyelinating plaques within the inferior frontal and temporal lobes, the central brain structures associated with olfactory processing.

*Corresponding author: Umberto Castiello,Department of General Psychology, University of Padua, via Venezia, 8, 35131, Padova, Italy,Tel: 0039-049-8276659; Fax: 0039-049-8276600, E-mail: umberto.castiello@unipd.it

Received September 04, 2010; Accepted October 12, 2010; Published Octobe 12, 2010

Citation: Parma V, Tornasi C, Grossi P, Atzori M, Perini P, et al. (2010) Specific Smell Dysfunctions in Relapsing-Remitting Multiple Sclerosis. J Neuro Neurophysiol 1:101. doi:10.4172/2155-9562.1000101

Copyright: ( $) 2010$ Parma V, et al. This is an open-access article distributed under the terms of the Creative Commons Attribution License, which permits unrestricted use, distribution, and reproduction in any medium, provided the original author and source are credited. 


\section{Methods \\ Participants}

Seventy-five female RRMS patients (age range 19 to 55 years; mean age $=37.5$ years; $\mathrm{SD}=8.7$ ) and 64 controls (age range 16 to 55 years; mean age $=35.5$ years; $S D=8.3$ ) were enrolled in the experiment (Table 1). For the MS group the disease duration ranged from 1-28 years (mean 9.22 years). All patients were selected by three board certified neurologists after having their diagnosis verified on the basis of the revised McDonald criteria [25] and who were regularly followed at the MS Centre of the Veneto Region. In order to evaluate the disability of the RRMS patients the Expanded Disability Status Scale (EDSS) [26] was administered $($ EDSS mean $=1.80 ;$ SD $=1.21$ ). Both patients and controls were tested with the Mini Mental State Examination (MMSE) test [27] (patients: MMSE mean $=29.28$; $\mathrm{SD}=$ 0.64; controls: MMSE mean $=29.73$; $S D=0.52$ ), the Beck Depression Inventory - II (BDI-II) [28] and the Beck Anxiety Inventory (BAI) [29] to exclude participants with cognitive impairment, depression and/ or anxiety at the time of olfactory testing. Patients treated with immunosuppressants, prone to epilepsy or undergoing steroid

\begin{tabular}{|c|c|c|c|c|}
\hline ID & Age & Onset yrs & DMA & MMSE \\
\hline 1 & 29 & 7 & IFN $\beta$ i.m. & 28 \\
\hline 2 & 28 & 6 & IFN $\beta$ i.m. & 28 \\
\hline 3 & 42 & 9 & IFN $\beta$ s.c. & 30 \\
\hline 4 & 41 & 5 & IFN $\beta$ s.c. & 29 \\
\hline 5 & 28 & 5 & - & 29 \\
\hline 6 & 29 & 3 & IFN $\beta$ i.m. & 29 \\
\hline 7 & 44 & 22 & - & 29 \\
\hline 8 & 42 & 14 & IFN $\beta$ s.c. & 29 \\
\hline 9 & 49 & 6 & IFN $\beta$ i.m. & 29 \\
\hline 10 & 39 & 8 & IFN $\beta$ i.m. & 29 \\
\hline 11 & 33 & 15 & - & 29 \\
\hline 12 & 40 & 5 & IFN $\beta$ i.m. & 29 \\
\hline 13 & 46 & 2 & - & 30 \\
\hline 14 & 30 & 5 & glatiramer acetate & 30 \\
\hline 15 & 44 & 8 & IFN $\beta$ i.m. & 30 \\
\hline 16 & 43 & 4 & IFN $\beta$ s.c. & 29 \\
\hline 17 & 52 & 15 & IFN $\beta$ s.c. & 29 \\
\hline 18 & 37 & 13 & - & 29 \\
\hline 19 & 55 & 13 & glatiramer acetate & 30 \\
\hline 20 & 33 & 7 & - & 30 \\
\hline 21 & 44 & 7 & IFN $\beta$ i.m. & 29 \\
\hline 22 & 39 & 14 & IFNß s.c. & 30 \\
\hline 23 & 27 & 9 & IFN $\beta$ i.m. & 28 \\
\hline 24 & 42 & 17 & glatiramer acetate & 29 \\
\hline 25 & 43 & 22 & IFN $\beta$ i.m. & 30 \\
\hline 26 & 20 & 2 & IFN $\beta$ i.m. & 29 \\
\hline 27 & 43 & 28 & 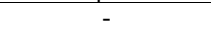 & 29 \\
\hline 28 & 29 & 11 & IFN $\beta$ s.c. & 30 \\
\hline 29 & 36 & 12 & IFN $\beta$ i.m. & 30 \\
\hline 30 & 46 & 3 & IFN $\beta$ s.c. & 30 \\
\hline 31 & 32 & 9 & - & 29 \\
\hline 32 & 36 & 2 & glatiramer acetate & 29 \\
\hline 33 & 33 & 10 & IFN $\beta$ i.m. & 29 \\
\hline 34 & 34 & 9 & IFN $\beta$ s.c. & 29 \\
\hline 35 & 30 & 3 & IFN $\beta$ i.m. & 30 \\
\hline 36 & 38 & 13 & IFN $\beta$ s.c. & 29 \\
\hline 37 & 37 & 7 & IFN $\beta$ i.m. & 28 \\
\hline 38 & 35 & 7 & IFN $\beta$ s.c. & 30 \\
\hline 39 & 52 & 16 & IFN $\beta$ i.m. & 28 \\
\hline 40 & 51 & 22 & IFN $\beta$ i.m. & 29 \\
\hline 41 & 37 & 20 & IFN $\beta$ s.c. & 30 \\
\hline 42 & 30 & 1 & IFN $\beta$ i.m. & 30 \\
\hline 43 & 27 & 1 & IFN $\beta$ s.c. & 30 \\
\hline 44 & 55 & 9 & glatiramer acetate & 29 \\
\hline 45 & 19 & 2 & IFN $\beta$ i.m. & 30 \\
\hline 46 & 42 & 6 & glatiramer acetate & 29 \\
\hline 47 & 23 & 6 & IFN $\beta$ s.c. & 30 \\
\hline 48 & 42 & 10 & IFN $\beta$ s.c. & 29 \\
\hline 49 & 31 & 2 & IFN $\beta$ i.m. & 30 \\
\hline 50 & 37 & 9 & IFN $\beta$ i.m. & 29 \\
\hline
\end{tabular}

DMA = Disease Modifyng Agent; IFN $\beta$ i.m = interferon beta into the muscle; IFN $\beta$ s.c = interferon beta subcutaneous.

Table 1 Demographical, pharmacological and cognitive preservation data for the 50 females RRMS patients participating in the study. therapy were excluded from the sample [30]. Finally, a questionnaire was administered to all participants to ascertain the previous history of nasal disease, smoking habits and the current status of olfactory functions. All patients and controls affirmed they were nonsmokers. On the basis of these exclusion criteria, 25 patients and 14 controls were excluded. The final cohort consisted of 50 female RRMS patients (mean age $=37.4$ years; $S D=8.7$; see Table 1 ) and 50 female age-matched controls (mean age $=35.5$ years; $S D=14.2$ ). All participants were naive as to the purpose of the investigation and gave informed written consent to participate in the study. The experimental procedures were approved by the Institutional Review Board at the University of Padova and were in accordance with the Declaration of Helsinki.

\section{The Sniffin' Sticks Extended Test}

To test the olfactory functions we used the Sniffin' Sticks Extended Test (Burghart Messtechnik GmbH, Wedel, Germany), a test devised to examine the three main aspects of olfactory functions, namely threshold, discrimination and identification, by means of pen-like odour dispensing devices presented alone (identification subtest) or in triplets (threshold and discrimination subtests) [21,22]. Normative data by age and gender for this test were used to determine the relative degree of general and specific olfactory loss which permitted a categorization of olfactory function in normal people and those with hyposmia and functional anosmia [31].

\section{Procedure}

The experimental session began with the collection of anamnestic data concerned with age, possible epilepsy and pharmacological history. Then the experimenter administered the 14-item questionnaire for testing the status of olfactory functions. During this phase participants were not allowed to eat and/or drink. Subsequently, the Sniffin' Sticks Extended Test was administered. Please refer to Kobal and colleagues [22] for more details regarding the procedure administration. The experimental session lasted approximately 60 minutes.

\section{Magnetic resonance images}

The MR images for a subgroup of the tested patients $(\mathrm{N}=13)$ were acquired within one month of administration of the olfactory test by means of a $1.5 \mathrm{~T}$ Philips Achieva (Philips Medical Systems, Best, Netherlands). The MR images were acquired on the same day as the Sniffin' Sticks test was performed for five of the patients. No major hardware upgrades were applied to the scanner during the study and quality evaluation sessions took place weekly to guarantee measurement stability. The following sets of images were acquired: (i) Fluid attenuated inversion recovery (FLAIR), a 2D sequence with 50 contiguous axial slices (TE $120 \mathrm{~ms}$, TR $10000 \mathrm{~ms}$, inversion time 2500 ms, slice thickness $3.0 \mathrm{~mm}$, matrix $256 \times 256$, gap 0); (ii) Turbo-spin echo Dp/T2 (TSE) sequences. By using a semiautomatic thresholding technique, implemented in the software called Medical Images Processing, Analysis and Visualization (MIPAV) (http://mipav.cit.nih. gov) developed at the National Institute of Health (NIH), lesions were selected and segmented on the FLAIR images providing a white matter T2 (WMT2) hyperintense lesion volume (T2LV). The presence of possible FLAIR-related artifacts was controlled on proton density and T2 images. In addition we used a digital version of the Talairach and Tournoux atlas [32] to perform regional volumetric analyses The number and the volume (expressed in $\mathrm{mm}^{3}$ ) of demyelinating plaques within the inferior frontal and inferior temporal lobes and within the whole brain, excluding the IFTL complex, were calculated by means of MR examination. These brain regions contain the major zones of known central olfactory connections and include, respectively, (i) 
the olfactory striae, subcallosal medial frontal lobe, paraterminal gyrus, orbitofrontal zone and gyrus rectus, and (ii) the prepiriform, enthorinal, amygdaloidal, hippocampal, and parahippocampal regions of the brain. The inferior frontal lobes were designated as being inferior and anterior to the body and genu of the corpus callosum, respectively. The inferior temporal lobes were considered as being superiorly bound by the plane of the Sylvian fissure. Plaques were counted and measured without knowledge of the scores obtained with the Sniffin' Sticks Extended Test by two independent expert neuroradiologists. A reliability test revealed no significant differences between the two neuroradiologists with respect to plaque counting.

\section{Statistical analyses}

The following descriptive measures were calculated: mean, standard deviation and the minimum and maximum values together with the percentiles (see Table 2). A Chi-square test was used to compare global olfactory performance in the RRMS group and the control group. A between-subjects MANCOVA together with Spearman's and Pearson's correlations were used as appropriate. The MANCOVA was applied to compare olfactory performance in the RRMS and control group, with 'group' as the independent variable and each of the olfactory indexes as dependent variables. 'Age' was used as the covariate to statistically control for variance in olfactory performance. Spearman's rank correlation coefficients were used to examine the relationship between olfactory Sniffin' Sticks scores, MRI data (plaque numbers and volume within and outside the IFTLs) and clinical variables (i.e., MS onset). Partial correlation analysis was performed to remove the effect of potential confounding factors.

\begin{tabular}{|c|c|c|c|c|c|c|c|c|c|}
\hline & & \multicolumn{4}{|c|}{ MS Patients } & \multicolumn{4}{|c|}{ Normative Data } \\
\hline & & THR & DIS & ID & TDI & THR & DIS & ID & TDI \\
\hline $\begin{array}{c}\text { AGE } \\
\text { GROUP }\end{array}$ & $16-35 \mathrm{yrs}$ & & & & & & & & \\
\hline $\mathrm{N}$ & & 25 & 24 & 25 & 24 & 760 & 741 & 827 & 704 \\
\hline Mean & & 8,44 & 12,17 & 13,33 & 33,94 & 9,39 & 12,91 & 13,68 & 36,06 \\
\hline SD & & 3,00 & 1,53 & 1,44 & 4,10 & 2,56 & 1,92 & 1,62 & 4,17 \\
\hline Minimum & & 3,50 & 10,00 & 10,00 & 27,30 & 1,75 & 5,00 & 8,00 & 23,00 \\
\hline Maximum & & 15,00 & 15,00 & 16,00 & 41,00 & 16,00 & 16,00 & 16,00 & 46,75 \\
\hline Percentiles & 5 & 3,69 & 10,00 & 10,25 & 27,34 & 5,51 & 9,00 & 11,00 & 29,50 \\
\hline & 10 & 4,75 & 10,00 & 11,50 & 28,22 & 6,50 & 10,00 & 11,00 & 30,50 \\
\hline & 25 & 6,38 & 11,00 & 12,00 & 30,30 & 7,50 & 12,00 & 13,00 & 33,50 \\
\hline & 50 & 7,63 & 12,00 & 13,50 & 33,80 & 9,00 & 13,00 & 14,00 & 36,00 \\
\hline & 75 & 10,75 & 14,00 & 14,00 & 37,00 & 11,25 & 14,00 & 15,00 & 39,00 \\
\hline & 90 & 13,00 & 14,00 & 15,00 & 40,30 & 12,50 & 15,00 & 16,00 & 41,50 \\
\hline & 95 & 14,50 & 14,80 & 15,75 & 40,90 & 14,00 & 16,00 & 16,00 & 43,00 \\
\hline $\begin{array}{c}\text { AGE } \\
\text { GROUP }\end{array}$ & $36-55$ yrs & & & & & & & & \\
\hline $\mathrm{N}$ & & 25 & 25 & 25 & 25 & 295 & 291 & 586 & 288 \\
\hline Mean & & 8,89 & 11,48 & 12,23 & 32,59 & 9,08 & 12,46 & 13,49 & 35,16 \\
\hline SD & & 2,54 & 1,85 & 1,56 & 3,65 & 3,09 & 1,96 & 1,56 & 4,52 \\
\hline Minimum & & 5 & 8,00 & 9,00 & 24,80 & 1,00 & 6,00 & 4,00 & 22,50 \\
\hline Maximum & & 15,00 & 15,00 & 15,00 & 39,00 & 16,00 & 16,00 & 16,00 & 45,75 \\
\hline Percentiles & 5 & 5,45 & 8,30 & 9,35 & 25,61 & 4,25 & 9,00 & 11,00 & 26,86 \\
\hline & 10 & 6,50 & 9,00 & 10,00 & 28,10 & 5,50 & 10,00 & 12,00 & 28,75 \\
\hline & 25 & 6,88 & 10,00 & 11,00 & 29,75 & 6,75 & 11,00 & 13,00 & 32,50 \\
\hline & 50 & 8,00 & 12,00 & 12,00 & 32,80 & 8,75 & 13,00 & 14,00 & 35,50 \\
\hline & 75 & 10,88 & 13,00 & 13,00 & 35,15 & 11,00 & 14,00 & 15,00 & 38,00 \\
\hline & 90 & 12,80 & 14,00 & 15,00 & 37,90 & 13,60 & 15,00 & 15,00 & 40,50 \\
\hline & 95 & 14,48 & 14,70 & 15,00 & 38,85 & 15,30 & 15,00 & 16,00 & 42,89 \\
\hline
\end{tabular}

THR = Threshold; DIS = Discrimination; ID = Identification; TDI = The sum of THR, DIS and ID.

Table 2: Sniffin' Sticks scores for the MS patients and the considered female normative population.

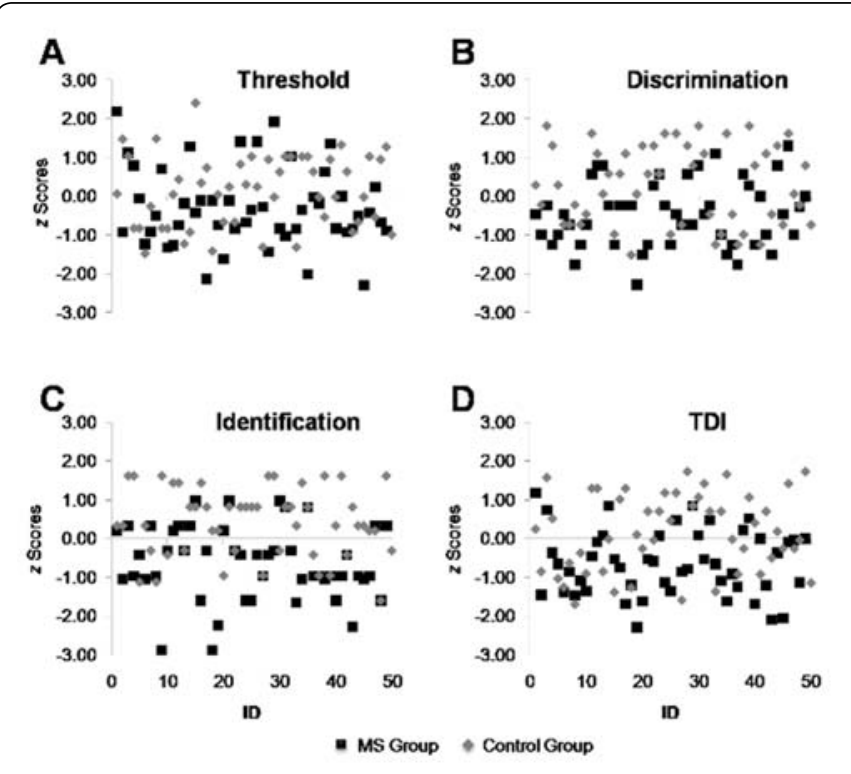

Figure 1: MS and control group Sniffin' Sticks scores expressed with standard deviations from the age- and sex-matched normative population means. Panel A represents threshold scores, Panel B represents discrimination scores, Panel $C$ represents identification scores and Panel D represents TDI scores.

\section{Results}

\section{Hyposmia and functional anosmia}

The Sniffin' Sticks Extended Test ascertains the presence of both hyposmia and functional anosmia. Hummel et al. [21] defines the TDI cut-off point between normosmic patients and hyposmic patients as 30.3. Following this criterion, we found a percentage of hyposmic participants of $34 \%$ (17 out of 50 ) within our RRMS group and of $8 \%$ (4 out of 50) within our control group. A Chi-square test revealed that hyposmic participants were statistically more frequent in the patient group than in the control group $\left(\chi^{2}=8.04, P<0.005\right)$.

With respect to functional anosmia, which refers to a TDI score less than $16.5[31,33]$, none of the RRMS or control participants were found to be totally functionally anosmic.

\section{Comparing RRMS and control group olfactory performance}

MANCOVA Wilks' lambda revealed a significant main effect for 'group' $\left(\mathrm{F}_{4,91}=239.3, P<0.0001, \eta^{2}=0.26\right)$. The covariate analyses for 'age' were significant $\left(\mathrm{F}_{4,91}=4.29, P<0.05, \eta^{2}=\right.$ 0.23). Because the MANCOVA revealed a significant main effect of group, examination of univariate ANCOVA analyses of the dependent variables was performed to identify which dependent variable contributed to the overall effect. Significant group effects emerged for the discrimination and identification scores $\left(P=0.01, \eta^{2}=0.10\right.$ and $P=0.0001, \eta^{2}=0.20$, respectively). No significant group effect was found when the threshold and TDI scores were considered $(P$ $\left.>0.05, \eta^{2}=0.05\right)$. Significant covariate relationships were also observed. Age was significant for both the identification and the TDI scores $\left(P<0.05, \eta^{2}=0.07\right)$. Pearson's correlations indicated that as age increased identification and TDI scores significantly decreased in both the RRMS and the control group $(r=-0.27, P<0.01)$.

\section{Correlating the duration of MS with olfactory functions and} neuropathological markers

As shown in Table 3, no significant correlation was found between 


\begin{tabular}{|c|c|c|c|c|c|c|c|c|}
\hline & \multicolumn{3}{|c|}{ Test olfactory scores } & \multicolumn{3}{c|}{ Plaques Number } & Plaques Volume \\
\hline & THR & DIS & ID & TDI & Within IFTL & Outside IFTL & Within IFTL & Outside IFTL \\
\hline Years onset & -0.10 & -0.14 & -0.22 & -0.22 & 0.04 & 0.19 & 0.25 \\
\hline
\end{tabular}

THR = Threshold; DIS = Discrimination; ID = Identification; TDI = The sum of THR, DIS and ID, IFLT = Inferior frontal and temporal lobes.

Table 3: Pearson's correlation between the years from the onset of MS, the Sniffin' Sticks scores and the neuropathological markers within and outside the IFTL complex.

\begin{tabular}{|c|c|c|c|c|}
\hline & \multicolumn{2}{|c|}{ Plaques Number } & \multicolumn{2}{|c|}{ Plaques Volume } \\
\hline & Within IFTL & Outside IFTL & Within IFTL & Outside IFTL \\
\hline Threshold & -0.48 & 0.04 & -0.36 & -0.09 \\
\hline Discrimination & -0.01 & -0.08 & -0.10 & -0.30 \\
\hline Identification & 0.37 & 0.22 & 0.15 & 0.07 \\
\hline TDI & -0.20 & 0.08 & -0.23 & -0.15 \\
\hline
\end{tabular}

$\mathrm{IFTL}=$ Inferior frontal and temporal lobes.

Table 4: Partial Correlation between the neuropathological marker and the Sniffin' Sticks scores controlling for age and years from MS onset.

the duration of MS and the scores obtained with the Sniffin' Sticks Extended Test, the number and the volume of the plaques within the IFTL complex (Table 3) and the areas outside the IFTL complex in terms of plaque numbers and plaque volumes (Table 3).

\section{Correlating the number and the volume of the plaques within and outside the IFTL complex with olfactory indexes}

Correlations between the number and the volume of the plaques within the IFTL complex, in the whole brain excluding the IFTL complex (e.g., outside IFTLs) and the olfactory indexes were performed. The number and volume of plaque within the IFTL complex did not negatively correlate either with any of the olfactory indexes considered or with the areas outside the IFTL complex in terms of plaque number and plaque volume. To remove the effect of potential confounders such as age and disease duration, partial correlations were also performed (Table 4). Thus, no association between Sniffin' Sticks scores and neuropathological markers within and outside the IFTL complex was demonstrated $(P>0.05)$. As an example of how the correlation between the number of plaques within the IFTL complex and the olfactory scores (i.e., TDI) might not be an optimal index, Figure 2 shows the MR images for two representative patients with the same sub-threshold performance $(\mathrm{TDI}=29$ ) on the olfactory tests, one with a small number of plaques (Figure 2A) and the other with a larger number (Figure 2B) of plaques.

\section{Discussion}

The main aim of the present study was to evaluate olfactory functions in a carefully selected group of female RRMS patients. Furthermore, we ascertained whether olfactory scores correlated with the number and volume of WMT2 lesions within (and outside) central eminent olfactory regions (IFTL complex).

Estimates of the prevalence of olfactory dysfunctions in MS vary widely $[5,6,8,11,20]$. Previous studies only testing odour
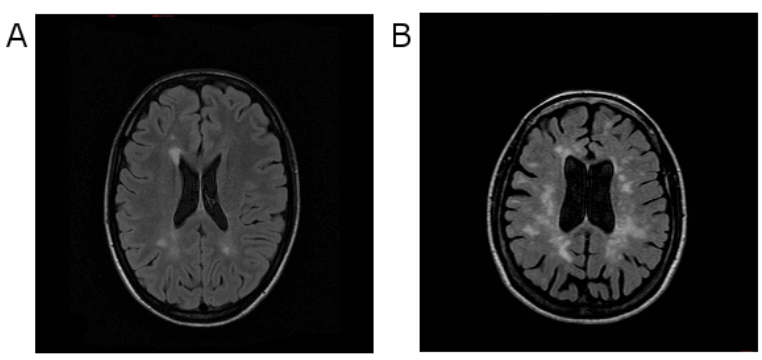

Figure 2: Panel A represents an axial T2-weighted MRI scan for a hyposmic patient (TDI =29), a 30-year-old woman with a three-year history of MS. Panel $B$ represents an axial T2-weighted MRI scan for a hyposmic patient $(T D I=29)$, a 39-year-old woman with an eight-year history of MS. identification on unspecified MS-subtypes samples reported olfactory dysfunctions affecting $15 \%[8], 35 \%$ and $38 \%[5,6,10]$ and $45 \%[9]$ of the considered MS populations. Various factors, such as the administered olfactory test, the patients' selection criteria (i.e., age, gender, clinical form of MS), the interval of time since the last relapse/highdose steroid therapy and concomitant medication affecting olfactory function (i.e., calcium-channel inhibitors, chemotherapeutics), might have contributed to these discrepancies. As an example, a study by Hawkes et al. [8] included patients who had experienced a recent relapse and received a course of high-dose steroids, which could have modified their performance either at the nose level or within the central olfactory pathways. In our study, $34 \%$ of the RRMS participants tested in our clinical setting exhibited a decreased olfactory ability on smell testing, which is in line with previous reports $[5,6,10]$ but, importantly, was obtained from a carefully selected group of RRMS patients: they were all females, had not received drugs known to affect the olfactory system, did not smoke and they were far from the last relapse/steroid course.

To the best of our knowledge, no previous research has evaluated specific aspects of olfactory functions in order to obtain detailed information on the smelling ability of MS patients. In this perspective, our findings not only support a general loss in the sense of smell of MS patients, but also suggest the presence of specific olfactory dysfunctions. When comparing the scores for the odour discrimination and identification tasks between the RRMS and the control group, olfactory deficits within the RRMS sample did emerge. The odour threshold scores did not significantly discriminate between the RRMS and the control group. Taken altogether these findings are in collusion with previous research reporting that odour identification ability was, to a certain extent, compromised in MS patients[5-10].

Another aspect of the present findings is that both the identification and the TDI scores seemed to be significantly affected by age-related effects. In other words, part of the amount of variance for the global and identification components of olfactory performance was accounted for by age, in the older participants, for both the MS and the control group, who performed worse than younger participants. Nevertheless, the significant MANCOVA results, considering 'age' as a covariate, suggested that the specific impairment in odour identification and general olfactory loss is a disease-related progression of the olfactory deficit.

In neural terms, we expected a strong negative correlation between the number of WMT2 lesions detected within the regions of the frontal and temporal lobes involved in olfaction and the scores obtained for the different components of the olfactory test $[6,7,19]$. Although we adopted a methodological approach similar 
to that of previous studies $[5-7,19]$, we failed to demonstrate such a relationship. This result was also found for the patients who had the olfactory assessment and the MR scan on the same day. This is important because it might well be that from the time the MR assessment was conducted to the time the olfactory test was administered the number and volume of the WMT2 lesions might have changed. We acknowledge that due to the limited number of patients who underwent MRI scanning we might not be able to state definite conclusions. Further investigation in highly homogeneous samples of patients is needed. However, it is worth mentioning that the studies which reported a strong correlation between the number of plaques and the UPSIT score considered an even smaller sample [7].

The finding of a lack of correlation between the decrease in olfactory performance and structural changes in central olfactory areas might suggest two alternative explanations. On the one hand, the olfactory dysfunctions in RRMS might be linked to a central functional rather than a structural impairment. To this end, recent research has outlined a diffuse brain network activating task-specific regions while performing different olfactory tasks [34]. Specifically, odour threshold is linked to the activation of right thalamus, amygdala-pyriform, cingulate, orbito-frontal and insular cortex whereas qualitative odour discrimination is known to engage thalamus, right caudate, subiculum, cingulate, orbito-frontal, pre-frontal, left insular and right cerebellar cortex [34]. Odour identification, though sharing part of the odour discrimination activation (thalamus, cingulate, orbitofrontal, pre-frontal, left insular and right cerebellar cortex), recruits, in addition, the pyriform cortex and sectors of the temporal and parietal cortex [34]. Given that an odour discrimination and identification loss is reported in our RRMS sample, it is tempting to speculate that the results of the present study might reflect the impairment of brain circuits engaged both in the odour discrimination and identification processes. Such an hypothesis seems to be supported by evidence from neurophysiological data considering the orbito- frontal cortex (OFC). As an example, Critchley and Rolls [35] demonstrated that the primate OFC responds in a highly selective manner to olfactory stimuli. In a similar vein, neuroimaging studies report that OFC lesions in the human brain account for specific smell disturbances, such as odour discrimination and identification impairment $[36,37]$.

On the other hand, olfactory dysfunctions in RRMS might reflect a peripheral rather than a central deficit. There may be the possibility that the odour spatial map is primarily affected by the disease. In the olfactory system a spatial map for odour detection already exists in the periphery [38], in which each olfactory neuron expresses just one of the 350 (in human) odorant receptors [39] and olfactory receptors also play an instructive role in determining the central projections of the olfactory neurons in which they are expressed [40]. Since each receptor responds to several odour molecules as well as each odour molecule is capable to stimulate a variety of receptors, the mammalian olfactory system uses a combinatorial receptor coding scheme to identify and discriminate odours [41]. Thus, an impairment of such combinatorial coding may explain the deficits in identification and discrimination of odours in MS. In other words, slight alterations of this wiring diagram might be responsible for an impairment of olfactory performance in MS.

With respect to the considered plaques indexes, not only we took into account the number of plaques, but we extended this literature by performing analysis of the volume of plaques within the IFTLs. At present, this measurement is considered to be less prone to the subjective judgment of the operator than the counting of plaques.
The plaque volume analysis did not find any significant correlations with the olfactory scores.

In this respect, it is of interest to draw a parallel between the present findings on RRMS patients and those obtained for people affected either by Parkinson's disease (PD) or degenerative ataxias. The extensive literature on olfactory disturbances in PD [42] also suggests that the olfactory disorder is a sensitive sign of pathology. However, as found here, the olfactory loss in PD is unspecific and it does not correlate with disease severity [43]. Furthermore, the present findings are remindful of those reported for degenerative ataxias patients, in which smell test scores do correlate neither with a genetic severity marker (GAA trinuclotide repeats) nor with disease duration [44].

Finally, the limitations of conventional MRI in depicting MS pathology may explain the discrepancies between the findings of the present study and previous ones. Several reviews and consensus statements over the past few years have questioned the value of current MR measures (e.g., T2 lesion burden, number of T2 lesions) as surrogate markers by noting that the correlation between these MRI measures and disability has been relatively poor $[45,46]$. Indeed, WMT2 lesion burden does not reflect the complexity of MS pathology, which includes cortical demyelinization and atrophy, spinal cord involvement and subtle biochemical alterations in the normally-appearing white matter $[47,48]$.

Our data confirms and extends previous findings on the evaluation of olfactory functions in MS patients. They outline the importance of using highly reliable tests able to capture more finelygrained aspects of olfactory performance together with the use of more carefully selected population samples. Although no correlations were identified between the olfactory scores and the neuropathological markers, we suggest that future research on this issue should consider less operator-dependent measures such as plaques volume. Moreover, it will be of great interest to explore if (and to which extent) MS patients share the same functional activations in brain circuits engaged in olfactory performance when compared to control participants. Furthermore, having insights on the molecular basis of the peripheral functioning in MS patients might help in clarifying the causal relationship of the olfactory impairment found in this population. Future research should also consider testing of the olfactory ability in pre-clinical, primary and secondary progressive homogeneous MS samples in order to elucidate the features of olfactory loss throughout the natural course of MS. This might open to the possibility for the identification of some markers, either functional or molecular, as to improve patients' outcomes. Since the sense of smell is fundamental for the quality of life, intended both in terms of survival mechanisms (e.g. detection and identification of potentially dangerous events signalled through smoke, the leaking of natural gas and spoiled foods) and in terms of daily well-being (e.g. appreciation of food, nutritional status, mood rate and social interactions), we suggest that a precise assessment of olfactory functions might be routinely performed in MS patients.

\section{References}

1. Confavreux C, Vukusic S, Moreau T, Adeleine P ( 2000) Relapses and progression of disability in multiple sclerosis. N Engl J Med 343: 1430-1438.

2. Sørensen TL, Frederiksen JL, Brønnum-Hansen H, Petersen HC ( 1999) Optic neuritis as onset manifestation of multiple sclerosis: a nationwide, longterm survey Neurology 53: 473-478.

3. Compston A (1994) The epidemiology of multiple sclerosis: principles, achievements, and recommendations. Ann Neurol 36: S211-S217. 
4. Constantinescu CS, Raps EC, Cohen JA, West SE, Doty RL (1994) Olfactory disturbances as the initial or most prominent symptom of multiple sclerosis. $J$ Neurol Neurosurg Psychiatry 57: 1011-1012.

5. Doty RL, Li C, Mannon LJ, Yousem DM ( 1998) Olfactory dysfunction in MS. Relation to plaque load in inferior frontal and temporal lobes. Ann N Y Acad Sci 855: 781-786

6. Doty RL, Li C, Mannon LJ, Yousem DM (1999) Olfactory dysfunction in multiple sclerosis: Relation to longitudinal changes in plaque numbers in central olfactory structures. Neurology 53: 880-882.

7. Doty RL, Li C, Mannon LJ, Yousem DM (1997) Olfactory dysfunction in multiple sclerosis N Engl J Med 336: 1918-1919.

8. Hawkes CH, Shephard BC, Kobal G (1997) Assessment of olfaction in multiple sclerosis: Evidence of dysfunction by olfactory evoked response and identification tests. J Neurol Neurosurg Psychiatry 63: 145-151.

9. Pinching AJ (1977) Clinical testing of olfaction reassessed. Brain 100: 377388.

10. Wender M, Szmeja Z (1971) Examination of the hearing, the vestibular system functions, taste and olfactory systems in patients with disseminated sclerosis. Neurol Neurochir Pol 5: 179-184.

11. Kesslak JP, Cotman CW, Chui HC, Van den Noort S, Fang H, et al. (1988) Olfactory tests as possible probes for detecting and monitoring Alzheimer's disease. Neurobiol Aging 2: 399-403.

12. Lumsden CE (1970) The neuropathology of multiple sclerosis; in Vinken PJ, Bruyn GW (eds): Handbook of clinical neurology. Amsterdam, Elsevier pp 217 309.

13. Samkoff LM, Alan J. Tuchman, Michael Daras, Barbara S. Koppel (1996) A Quantitative Study of Olfaction in Multiple Sclerosis. Neurorehabil Neural Repair 10: 97-99.

14. Doty RL, Frye R (1989) Influence of nasal obstruction on smell function. Otolaryngol Clin North Am 22: 397-411.

15. Doty RL, McKeown DA, Lee WW, Shaman P (1995) A study of the test-retest reliability of ten olfactory tests. Chem Senses 20: 645-656.

16. Doty RL, Shaman P, Applebaum SL, Giberson R, Siksorski L, et al. (1984) Smell identification ability: changes with age. Science 226: 1441-1443.

17. Kostic J, Stefanova E, Pekmezovic T, Drulovic J (2009) Correlation between olfactory dysfunction and linical parameters in patients with multiple sclerosis. Vojnosanit Pregl 66: 203-206.

18. Zivadinov R, Zorzon M, Monti Bragadin L,Pagliarto G, Cazzato G (1999) Olfactory loss in multiple sclerosis. J Neurol Sci 168: 127-130.

19. Zorzon M, Ukmar M, Bragadin LM, Zanier F, Antonello RM, et al.(2000) Olfactory dysfunction and extent of white matter abnormalities in multiple sclerosis: A clinical and MR study. Mult Scler 6: 386-90.

20. Ansari KA (1976) Olfaction in multiple sclerosis. With a note on the discrepancy between optic and olfactory involvement. Eur Neurol 14: 138-145.

21. Hummel T, Sekinger B, Wolf SR, Pauli E, Kobal G (1997) "Sniffin' Sticks": olfactory performance assessed by the combined testing of odour identification, odour discrimination and olfactory threshold. Chem Sens 22: 39-52.

22. Kobal G, Hummel T, Sekinger B, Barz S, Roscher S, et al. (1996) "Sniffin" Sticks": screening of olfactory performance. Rhinology 34: 222-226.

23. Albrecht J, Anzinger A, Kopietz R, Schopf V, Kleemann AM, et al. (2008) TestRetest Reliability of the Olfactory Detection Threshold Test of the Sniffin' Sticks. Chem Sens 33: 461-467.

24. Huijbregts SC, Kalkers NF, de Sonneville LM, de Groot V, Reuling IE, et al. (2004) Differences in cognitive impairment of relapsing remitting, secondary, and primary progressive MS. Neurology 63: 335-339.

25. McDonald WI, Compston A, Edan G, Goodkin D, Hartung HP, et al.(2001) Recommended diagnostic criteria for multiple sclerosis: Guidelines from the internatioal panel on the diagnosis of multiple sclerosis. Ann Neurol 50: 121-127.
26. Kurtzke JF (1983) Rating neurologic impairment in multiple sclerosis: an expanded disability status scale (EDSS). Neurology 33: 1444-1452.

27. Folstein MF, Folstein SE, McHugh PR (1975) "Mini-mental state". A practica method for grading the cognitive state of patients for the clinician. J Psychiatr Res 12: 189-198.

28. Beck AT, Steer RA, Brown GK. Manual for the Beck Depression Inventory-II. Psychological Corporation, San Antonio, USA, 1996.

29. Beck AT, Steer RA. Manual for the Beck Anxiety Inventor. Psychological Corporation, San Antonio, USA, 1990.

30. Stenner M, Vent J, Huttenbrink KB, Hummel T, Damm M (2008)Topical therapy in anosmia: relevance of steroids-responsiveness. Laryngoscope 118: 16811686.

31. Hummel T, Kobal G, Gudziol H, Mackay-Sim A (2007) Normative data for the "Sniffin' Sticks" including tests of odor identification, odor discrimination, and olfactory thresholds: An upgrade based on a group of more than 3,000 subjects. Eur Arch Otorhinolaryngol 264: 237-243.

32. Talairach J, Tourneaux P. Co-planar stereotaxic atlas of the human brain. Stuttgart, Thieme, 1988.

33. Kobal G, Klimek L, Wolfensberger M, Gudziol H, Temmel A, et al. (2000) Multicenter investigation of 1,036 subjects using a standardized method for the assessment of olfactory function combining tests of odour identification, odour discrimination, and olfactory thresholds. Eur Arch Otorhinolaryngol 257: 205211.

34. Savic I (2005) Brain imaging studies of the functional organization of human olfaction. Chem Senses 30: i222-i223.

35. Critchley HD, Rolls ET (1996) Olfactory neuronal responses in the primate orbitofrontal cortex: analysis in an olfactory discrimination task. J Neurophysiol 75 1659-1672.

36. Jones-Gotman M, Zatorre RJ (1988) Olfactory identification deficits in patients with focal cerebral excision. Neuropsychologia 26: 387-400.

37. Zatorre RJ, Jones-Gotman M (1991) Human olfactory discrimination after unilateral frontal or temporal lobectomy. Brain 114: 71-84.

38. Mombaerts P, Wang F, Dulac C, Chao SK, Nemes A, et al. (1996) Visualizing an olfactory sensory map. Cell $87: 675-686$.

39. Buck LB (2004) Olfactory receptors and odor coding in mammals. Nutr Rev 62 S184-S188.

40. Wang F, Nemes A, Mendelsohn M, Axel R (1998) Odorant receptors govern the formation of a precise topographic map. Cell 93: 47-60.

41. Malnic B, Hirono J, Sato T, Buck LB (1999) Combinatorial receptor codes for odors. Cell 96: 713-723.

42. Müller A, Müngersdorf M, Reichmann H, Strehle G, Hummel T (2002) Olfactory function in Parkinsonian syndromes. J Clin Neurosci 9: 521-524.

43. Doty RL (2007) Olfaction in Parkinson's disease. Parkinsonism. Relat Disord 3 S225-S228.

44. Connelly T, Farmer JM, Lynch DR, Doty RL (2003) Olfactory dysfunction in degenerative ataxias. J Neurol Neurosurg Psychiatry 74: 1435-1437.

45. Nyul LG, Udupa. JK (1999) On standardizing the MR image intensity scale. Magn Reson Med 42: 1072--1081.

46. Udupa JK, Nyul LG, Ge Y,Grossman RI (2001) Multiprotocol MR image segmentation in multiple sclerosis: experience with over 1,000 studies. Acad Radiol 8: 1116-1126.

47. Barkhof $F$ (2002) The clinico-radiological paradox in multiple sclerosis revisited Curr Opin Neurol 15: 239-245.

48. Miller DH, Grossman RI, Reingold SC, McFarland HF (1998) The role of magnetic resonance techniques in understanding and managing multiple sclerosis. Brain 121: 3-24 